\title{
Variations in the Left Coronary Artery
}

\author{
Sultana Ruma Alam ${ }^{*}$ \\ ${ }^{1}$ Department of Anatomy \\ University of Science \& Technology Chittagong (USTC) \\ Chittagong, Bangladesh.
}

\begin{abstract}
Background : There is a large spectrum of variations in the disposition of coronary arteries. Many of these variations are 'normal' and not considered as

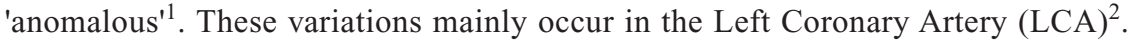
While some of these are benign and have no clinical consequences, other variants can cause important clinical manifestations including sudden death of the individual $^{3}$. Lack of knowledge of such variations can pose difficulties in percuteneous coronary arteriography, coronary artery bypass surgery or prosthetic valve replacement. A cadaveric study in unsuspected population can help to understand the variations that will be useful to determine the prevalence of certain variations. Thus the objective of this study was to analyze the characteristics of LCA that may be used in the diagnosis and treatment of its pathologies. Methods: The study was carried out in the Department of Anatomy, Chittagong Medical College (CMC) Chittagong over a period between Jan 2012 to Dec 2013 with ethical clearance. A detailed dissection of LCA and its branches in 50 cadaveric human hearts, fixed in $10 \%$ formalin was carried out to study normal and variant anatomy of LCA. The length of the main stem of LCA was measured by slide calipers. Results: The LCA was found to arise from the Left Posterior Aortic Sinus (LPAS) of the ascending aorta in $100 \%$ cases. The level of the ostia (Opening of coronary artery) was above the free margin of the aortic cusps in $98 \%$ cases. In all samples ostia were present below sinutubular ridge (A slight circumferential thickening separating bulbar aortic sinus and proximal ascending aorta). The length of the main stem of LCA (From origin to the point of termination into main branches) was found to range from $0.5-2 \mathrm{~cm}$. The LCA showed bifurcation in $74 \%$, trifurcation in $26 \%$ of cases. Left Anterior Descending artery (LAD) was found to terminate at the apex of the heart in $68 \%$ and at the posterior interventricular groove in 32\% cases. The Left Circumflex artery (LCx) was terminated at the crux of the heart in 52\%, near the crux in $44 \%$ and by crossing the crux in $4 \%$ cases. The Left Marginal Artery (LMA) which was present only in $34 \%$ cases, found to terminate nearer to the apex of the heart. $0 \%$ Left dominance of heart was observed. Conclusion: Simple attention to potential variations in the origin, number, level of ostia, length of the main stem, branching pattern, termination and distribution of LCA can greatly enhance clinical outcomes.
\end{abstract}

Key words: LCA; LAD; LCx; LMA; Variation.

\section{Dr. Sultana Ruma Alam}

Assistant Professor

Department of Anatomy

University of Science \& Technology Chittagong (USTC) Chittagong, Bangladesh.

Mobile : +8801716823953

Email :rumalam@hotmail.com

www.banglajol.info/index.php/CMOSHMCJ 


\section{INTRODUCTION}

The coronary arterial tree is designed specially to carry oxygen and nutrient rich blood directly to the heart muscle which requires energy to perform its work ${ }^{4}$.

Coronary arteries are the greatly enlarged vasa vasora running just deep to the epicardium ${ }^{3}$. The arterial tree supplies the heart by two coronary arteries ie. right and left coronary arteries which are the only branches of ascending aorta $^{4}$. Among these two the LCA shows a significant variation in its morphology with regard to origin, number, level of ostium, length of main stem, number of branches of its trunk, termination of its branches and distribution than the Right Coronary Artery (RCA) ${ }^{5}$. The knowledge of such variations of LCA is particularly important in determining areas related to occlusive arterial diseases, in hemodynamic procedures, in handling heart trauma and finally in terms of academic value.

\section{MATERIALS AND METHODS}

50 adult human cadaveric hearts were collected from Department of Anatomy, Chittagong Medical College (CMC) and samples were fixed in $10 \%$ formalin solution. After removal of epicardial adipose tissues, the visceral pericardium was removed. The LCA and its branches were dissected (As far as practicable) in the atrioventricular and interventricular grooves on the surface of the heart. The ascending aorta was cut longitudinally and then transversely to see the position and number of coronary ostia. Then the length of the main stem was measured with the help of slide calipers.

\section{RESULTS}

The ostium for Left Coronary Artery (LCA) was found in the left posterior aortic sinus of ascending aorta in all specimens. The ostium was above the free margins of aortic cusps within the sinus of Valsalva (The bulbous sinus and three aortic cusps) in 98\% samples. Length of main stem of LCA (From origin to the point of termination into main branches) was found to range from $0.5-2 \mathrm{~cm}$. The LCA was found to terminate at the junction between anterior interventricular sulcus and left anterior part of coronary sulcus. Here the artery was found to bifurcate in $74 \%$ and trifurcate in $26 \%$ cases. No quadrifurcation or pentafurcation was found. When bifurcated, the branches of LCA are Left Anterior Descending artery (LAD) and Left Circumflex (LCx) artery. When trifurcated, additional diagonal (Also called Ramus Intermidius) branch found to arise from angle between LAD and LCx. Left Marginal Artery (LMA) was present in 34\% cases and when present they were found to be branch of LCA in $100 \%$ cases. The present series of 50 hearts, $100 \%$ right dominance of coronary artery was found as because the posterior descending artery was branch of RCA in all samples. [The dominant coronary artery is that which gives the Posterior Descending (PDA) branch, traversing the posterior interventricular groove ${ }^{4}$.

\section{DISCUSSION}

The anatomy of LCA and its variations has recently been reemphasized in association with the use of coronary arteriography ${ }^{5}$. The importance of ostium anomalies relates to the surgical difficulties encountered in cannulating these vessels during open aortic surgery or in performing coronary arteriography ${ }^{5}$. The origin, number, location and size of the ostium are very important in performing successful performance of coronary angiogram.

The origins of the coronaries show great variability ${ }^{8}$. Seperate origin of LAD \& LCx in LPAS (Figure 1) LAD \& LCx arising from RCA, LAD arising from LPAS and LCX arising from RCA, absence of LCx artery, LCx originating from RCA or right sinus of Valsalva (Figure 2) LCA arising from right sinus of Valsalva, ectopic origin of LCA from pulmonary trunk or common carotid artery are some variations that have been documented by Bergman ${ }^{2}$.

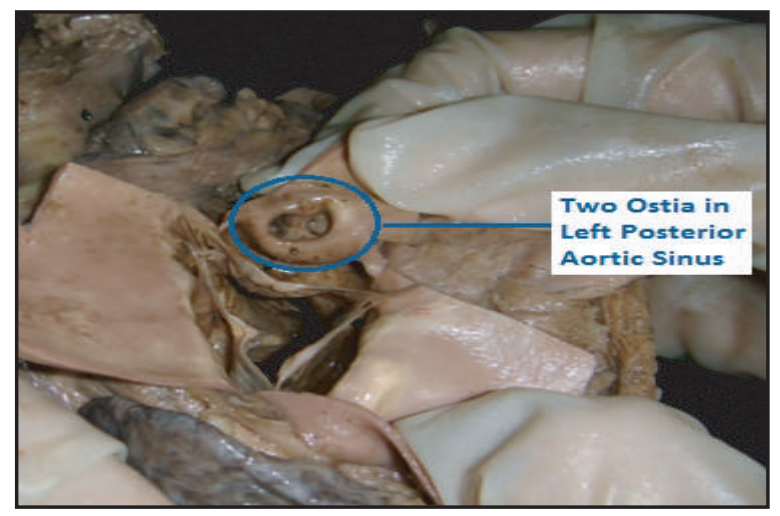

Figure 1 : Superior view of heart specimen showing two ostia in left posterior aortic sinus ${ }^{7}$.

When the LCx originates from the right sinus of Valsalva and follows a retro aortic course, there is the possibility that it might be damaged by sutures placed in the mitral annulus during mitral valve replacement or annuloplasty, resulting in infarction $^{8}$

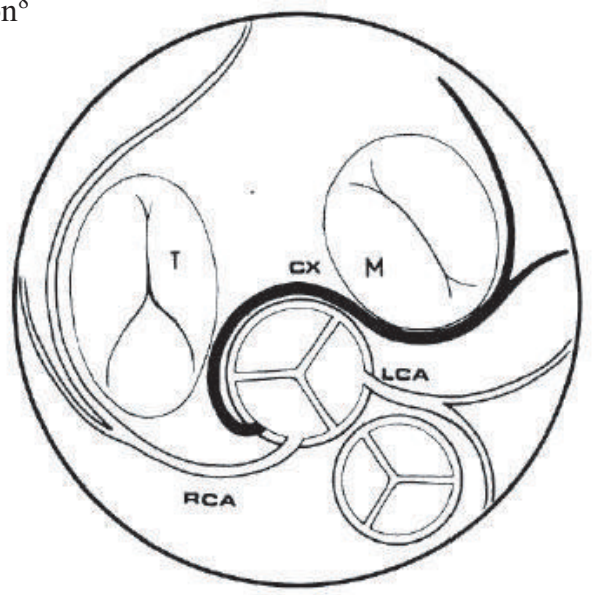

Figure 2 : Anomalous origin of the LCx artery from anterior aortic sinus ${ }^{8}$. Here $\mathrm{M}=$ Mitral valve, $\mathrm{T}=$ Tricuspid valve, $\mathrm{RCA}=$ Right Coronary Artery, $\mathrm{LCA}=$ Left Coronary Artery, $\mathrm{CX}=$ Circumflex artery. 
LCA arising from right sinus of Valsalva or from pulmonary trunk are potentially serious coronary anomalies which have been associated with myocardial ischaemia, ventricular dysfunction and sudden death ${ }^{2}$. In present study, the LCA was found to take origin from LPAS in all specimens which support the earlier studies by different authors $3,6,7,9,10,11,12,13,14$.

Multiple ostia in the LPAS is seen when LAD and LCX originate separately. The knowledge of presence of multiple coronary ostia is important. While performing coronary arteriography and angiography, a preliminary aortic root injection of the dye must be given to locate them so that the adverse outcomes of the procedure can be prevented ${ }^{7}$.

Normally, the coronary ostia are located above the free margins of the aortic cusps within the sinus of Valsalva and below sinutubular ridge $e^{1,5,6}$. This location of ostium below sinutubular ridge is important for maximum coronary diastolic filling ${ }^{7}$. In present study, the positioning of ostium of LCA was above the free margin of the aortic cusps in $98 \%$ and below the free margin of the cusps in $2 \%$ cases. But in all samples LCA ostium was found to present below the sinutubular ridge. Dombe (2012) Basir(1988) Kalpana (2003) and Shilpa mentioned the ostium to be present below sinutubular ridge in about $80 \%$ cases $^{5,9,13,15}$ (Table 1).

Table 1: Comparison of incidences of various locations of ostium of LCA in different studies.

\begin{tabular}{|c|c|c|}
\hline Author (s) & $\begin{array}{l}\text { Location of } \\
\text { ostium }\end{array}$ & $\begin{array}{l}\text { Incidence of } \\
\text { origin of LCA }\end{array}$ \\
\hline \multirow[t]{3}{*}{ Present study } & Sinus & $100 \%$ \\
\hline & Sinuaortic & - \\
\hline & Aortic & - \\
\hline \multirow[t]{3}{*}{ Basir $^{9}$} & Sinus & $100 \%$ \\
\hline & Sinuaortic & - \\
\hline & Aortic & - \\
\hline \multirow[t]{3}{*}{ Dombe $^{5}$} & Sinus & $80 \%$ \\
\hline & Sinuaortic & $17 \%$ \\
\hline & Aortic & $3 \%$ \\
\hline \multirow[t]{3}{*}{ Shilpa ${ }^{15}$} & Sinus & $80 \%$ \\
\hline & Sinuaortic & - \\
\hline & Aortic & $20 \%$ \\
\hline \multirow[t]{3}{*}{ Kalpana $^{13}$} & Sinus & $80 \%$ \\
\hline & Sinuaortic & - \\
\hline & Aortic & $20 \%$ \\
\hline \multirow[t]{3}{*}{ Mutyal $^{7}$} & Sinus & $68 \%$ \\
\hline & Sinuaortic & $18 \%$ \\
\hline & Aortic & $14 \%$ \\
\hline \multirow[t]{3}{*}{ Joshi $^{6}$} & Sinus & $80 \%$ \\
\hline & Sinuaortic & $15 \%$ \\
\hline & Aortic & $5 \%$ \\
\hline
\end{tabular}

Banchi (1964) reported the location of the ostium of LCA to be present below the free margins of the cusps in $18 \%$ cases $^{16}$. Furthermore, it was reported that the location of the ostium of LCA can be at or above the level of sinutubular ridge $^{5}$ (Figure 3 ).

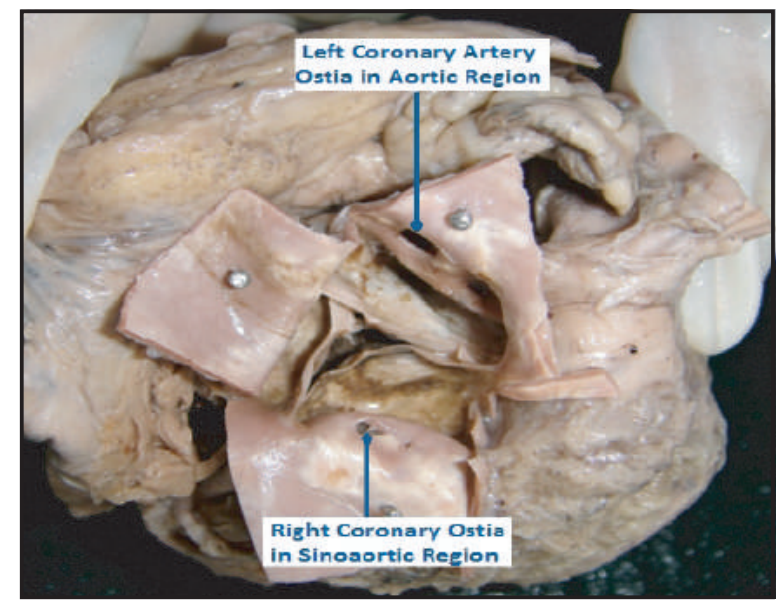

Figure 3 : Superior view of heart showing location of ostium of RCA below sniutubular ridge and ostium of LCA above sinutubular ridge ${ }^{5}$.

Difficulty in manipulating catheter tips will be considerably higher in patients with ostium level above the sinutubular $\operatorname{ridge}^{7}$ (Figure 4).

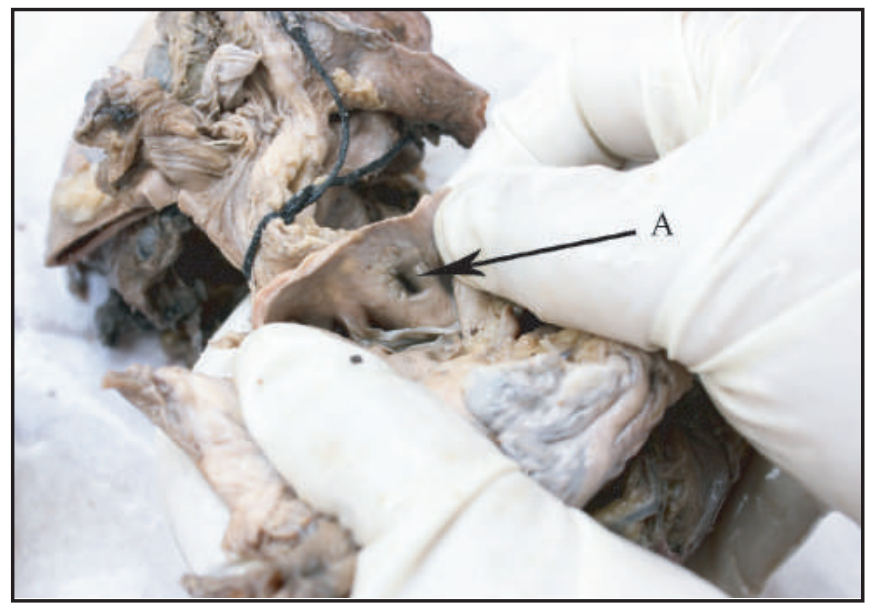

Figure 4 : A. Ostia of left coronary artery above free margin of the aortic cusps. Here A indicates the position of ostium of LCA.

High takeoff origins are likely to be missed during the procedures like coronary angiography if not borne in mind. Location of ostia above sinutubular ridge may decrease the coronary perfusion ${ }^{7}$. Such arteries can be at risk of being clamped during the valve replacement surgery with low clamping of Aorta. 
Usually length of LCA varies from few $\mathrm{mm}$ to few $\mathrm{cm}$. In the present study the length of the main stem of LCA was found to range from $0.5-2 \mathrm{~cm}$. Table 2 shows the comparison of average length of the main stem of LCA found in present study versus other studies. The findings are almost similar to the findings reported in various studies.

Table 2 : Comparison of length of main stem of LCA reported in different studies.

\begin{tabular}{lc} 
Author (s) & $\begin{array}{c}\text { Average length of } \\
\text { main stem of LCA }\end{array}$ \\
\hline Present study & $0.5-2 \mathrm{~cm}$ \\
Basir $^{7}$ & $0.5-1.8 \mathrm{~cm}$ \\
Kalpana $^{11}$ & $0.2-2 \mathrm{~cm}$ \\
Fiss $^{1}$ & $0.2-1.2 \mathrm{~cm}$ \\
Mutyal $^{5}$ & $0.5-2 \mathrm{~cm}$ \\
\hline
\end{tabular}

Variation in the length particularly of LCA is very important during coronary angiography. If the main trunk is short, the image of coronary arterial tree would be incomplete ${ }^{5}$. Because when catheter is inserted into one of the terminal branches, the opacification of the other branch does not occur and an incomplete image of coronary tree is seen ${ }^{5}$. During aortic valve surgery myocardial perfusion depends on the placement of one or more cannulas in the coronary arteries. In this regard length of LCA prior to bifurcation is particularly important. Short length of LCA may be predisposing factor to coronary arterial disease ${ }^{3}$.

The termination of LCA varies between two or three branches with the most common pattern bifurcation ${ }^{5}$. Other variations are trifurcation, quadrifurcation and pentafurcation. In present study LCA was found to bifurcate in $74 \%$ (Figure 5) and trifurcate in 26\% cases (Figure 6). No quadrifurcation or pentafurcation found. When bifurcated, the branches of LCA are Left Anterior Descending artery (LAD) and Left Circumflex (LCx) artery. When trifurcated, an additional diagonal (Also called Ramus Intermidius) branch found to arise from angle between $\mathrm{LAD}$ and $\mathrm{LCx}^{1}$.

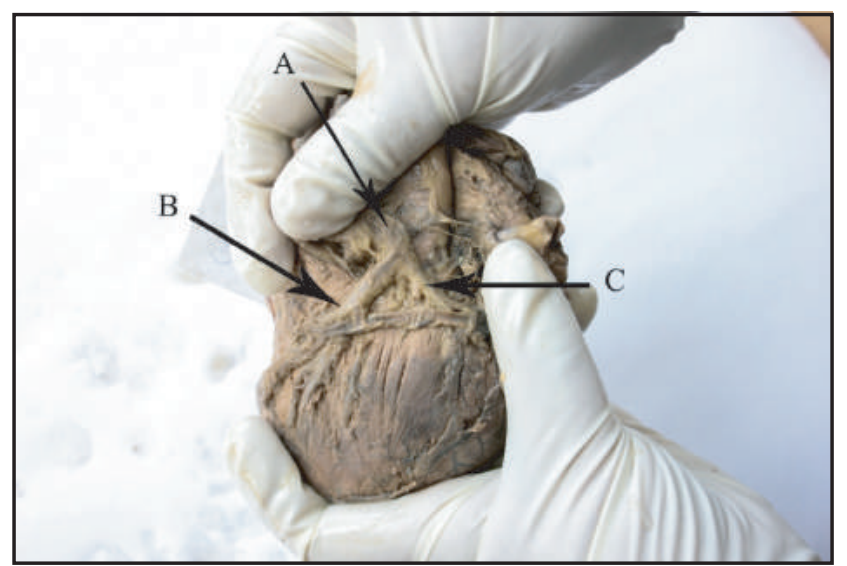

Figure 5 : Shows bifurcation of LCA. A) Left coronary artery B) Left anterior descending artery C) Left circumflex artery.

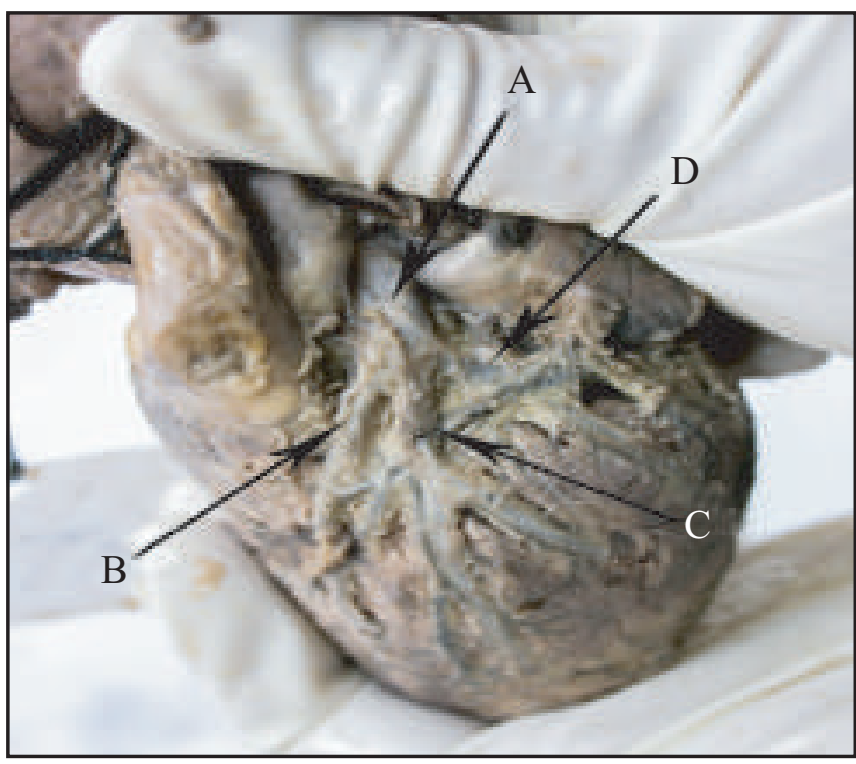

Figure 6 : Shows trifurcation of LCA. A) Left coronary artery with trifurcation B) Left anterior descending artery C) Diagonal artery D) Left circumflex artery.

Table 3 : Shows comparison of incidences of branching pattern of LCA in different studies.

\begin{tabular}{lcccc} 
Author (s) & \multicolumn{4}{c}{ Branching pattern of LCA } \\
& Bi & Tri & Quadri & Penta \\
\hline Present study & $74 \%$ & $26 \%$ & - & - \\
Basir $^{9}$ & $72 \%$ & $20 \%$ & $8 \%$ & - \\
Rahman $^{11}$ & $53 \%$ & $33 \%$ & $14 \%$ & - \\
Sarkar $^{10}$ & $47 \%$ & $45 \%$ & $6 \%$ & $2 \%$ \\
Hossain $^{12}$ & $50 \%$ & $44 \%$ & $6 \%$ & - \\
Dombe $^{5}$ & $55 \%$ & $36 \%$ & $8 \%$ & - \\
Kalpana $^{13}$ & $47 \%$ & $40 \%$ & $11 \%$ & $1 \%$ \\
\hline
\end{tabular}

Here, $\mathrm{Bi}=$ Bifurcation, Tri $=$ Trifurcation, Quadri= Quadrifurcation, Penta= Pentafurcation.

Other reported studies also show high incidences of bifurcation similar to present study. But other studies have reported higher incidences of trifurcation and quadrifurcation than the present study. Sarkar and Kalpana documented incidence of pentafurcation ${ }^{10,13}$ (Table 3 ). Identification of Ramus intermidius is very important clinically $^{5}$. Its area of distribution to the heart is small. In the absence of this artery the area is irrigated by the branches of LAD and LCx, so in occlusion of LAD and LCx more area is affected. When this ramus intermidius branch is present, its origin is frequently the site of narrowing 5 . 
Present study shows the LAD to terminate at the apex in $68 \%$ and in the posterior interventricular groove in $32 \%$ cases. Basir, Sarkar, Rahman and Hossain reported the artery to terminate in the posterior interventricular groove in $80 \%, 71 \%, 85 \%$ and $86 \%$ cases respectively ${ }^{9,10,11,12}$. In present study the LCx was found to terminate at the crux of the heart in $52 \%$, near the crux in $44 \%$ and by crossing the crux in $4 \%$ cases. Other studies by Basir, Sarkar and Rahman show that the LCx in majority cases terminated near the crux of the heart $(76 \%, 70 \%$ and $67 \%$ respectively $)^{9,10,11}$. The difference of results with other authors may be due to small sample size of present study. The LMA when present, found mostly to terminate near the apex of the heart.

The origin of PDA from RCA is the commonest anatomy in man and referred to as right dominance of heart. When $\mathrm{LCX}$ continues in the posterior interventricular groove as PDA then it is called left dominance of heart. Where the PDA is bilateral or absent it is said balanced circulation ${ }^{4}$. In $100 \%$ cases the present study showed right dominance of heart. Whereas a good percentage of left dominance of heart have been documented by several authors as mentioned in Table 4. As shown in table 4 Sarkar and Rahman and have also reported balanced circulation ${ }^{10,11}$.
Table 4 : Shows comparison of incidence of dominance of heart in different studies.

\begin{tabular}{lccc} 
Author (s) & \multicolumn{3}{c}{ Dominance pattern } \\
& Right & Left & Balanced \\
\hline Present study & $100 \%$ & - & - \\
Basir $^{9}$ & $84 \%$ & $16 \%$ & \\
Rahman $^{11}$ & $70 \%$ & $17 \%$ & $13 \%$ \\
Sarkar $^{10}$ & $63 \%$ & $9 \%$ & $28 \%$ \\
Hossain $^{12}$ & $90 \%$ & $10 \%$ & - \\
Kalpana $^{13}$ & $89 \%$ & $11 \%$ & - \\
\hline
\end{tabular}

\section{CONCLUSION}

Coronary arteries show many variations in branching and termination. All these are variations of "normal" that are not considered as "anomalous". But lack of knowledge of these variations may have dire consequences in catheterization, valve replacement, or bypass surgery ${ }^{2}$. So, further study on the relationship between the distribution of coronary artery and the most common areas of Myocardial Infarction as well as potential relationship between a special variation in arterial distribution and certain myocardial diseases is needed.

\section{ACKNOWLEDGEMENT}

1. Dr. Kazi Rafiqul Haque, Professor and Head, Department of Anatomy, University of Science \& Technology Chittagong (USTC) Chittagong.

2. Dr. Md. Ashrafuzzaman, Professor and Head, Department of Anatomy, Chittagong Medical College, Chittagong.

\section{DISCLOSURE}

The author declared no competing interest. 


\section{REFERENCES}

1. Fiss DM. Normal Coronary Anatomy and Anatomic Variations. Journals. 2007;36.

2. Bergman RA, Afifi AK, Miyanchir. Illustrated Encyclopedia of Human Anatomic Variation: Opus II: Cardiovascular system: Arteries: Head, Neck and Thorax- Coronary arteries. Anatomy Atlas.

3. Kulkarni JP. Variant anatomy of coronary arteries. Heart India. 2013; 46-51.

4. Allwork SP. The applied anatomy of the arterial blood supply to the heart in man. J Anat. 1987;153:1-16.

5. Dombe DD, Anitha T, Giri PA, Dombe SD, Ambiye MV. Clinically relevant morphometric analysis of Left coronary artery. International Journal of Biological and Medical research. 2012;3(1):1327-1330.

6. Joshi SD, Joshi SS, Athavalle SA. Origin of coronary arteries and their significance. Clinics (Saopaulo). 2010;65(1):79-84.

7. Mutyal SR, Mandke MD, Ambhiye MV. Study of Anatomical variation in origin of coronary arteries. International Journal of Biological and medical research. 2014;5(1):3802-3806.

8. M. Trivellato, M D, Paolo Angelini, MD, and Robert D. Leachman, M.D. Variations in coronary artery anatomy: Normal versus abnormal. Cardiovascular Disease, Bulletin of the Texas heart institute. 1980;7: 357-370.

9. Basir KE. A study of patterns and distributions of coronary arteries in adult postmortem human hearts in Bangladesh. M.Sc. thesis. University of Dhaka. 1988.

10. Sarker MSA. An anatomical study of the coronary arteries and the arch of the aorta in adult Bangladeshi people. M. Phil thesis. University of Dhaka. 1996.

11. Rahman ASMH. Anatomy of coronary circulation and dimensions of adult postmortem human hearts in Bangladesh. M. Phil thesis University of Dhaka. 1998.

12. Hossain MA. Postmortem study on principal branches of the coronary arteries in adult Bangladeshi people. M. Phil thesis. University of Dhaka. 2006

13. Kalpana R. A study on principal branches of coronary arteries in human. J Anat Soc India. 2003;52(2):137-140.

14. Gajbe UL, Gosani S, Meshram S, Gajbhiye VM. The anomalous origin of multiple coronary ostia and their clinical significance. Journal of Clinical and diagnostic research. 2010;4(1):2129-2133.

15. Shilpa B, Hukkeri VB, Potturi VB. A study of Variation anomalies of coronary artery in Cadaveric Human Hearts.

16. Banchi Cited in Blake HA, Manion WC, Mattingly TW, Baroldi G. Coronary artery anomalies. Circulation. 1964;30:927-937. 